secondary surgery (further FSS in 34). At univariate analysis, significant predictors of relapse were: size of largest extraovarian lesion, peritoneal cancer index, completeness of cytoreduction, type of implants. After multivariable analysis, the size of extra-ovarian lesions and the presence of invasive implants resulted as the only independent predictors of recurrence (Tab. 1). Median DFS and DSS were respectively 96 months (95\% CI, 17.5-174.4) and 290.4 months (95\% CI, 280.8-299.9) (figure 1). Thirty-one patients attempted to conceive: $23(74.2 \%)$ achieved at least one pregnancy and 20 $(64.5 \%)$ gave birth to a healthy child. At the end of the observation period, 96 patients (95\%) showed no evidence of disease, $2(2 \%)$ were alive with disease, and 3 patients (3\%) died from BOT.

Conclusions Despite the recurrence high rate, the survivals and pregnancy outcomes indicate that FSS could be considered in advanced BOTs. Among predictors of recurrence, oophorectomy (vs. cystectomy) has resulted not significant as in earlystage BOTs likely due to the advanced-stage setting. Size of extra-ovarian lesions and presence of invasive implants were the only significant predictors. Completeness of cytoreduction was lacking significance likely because of low number of patients with residual disease.

Disclosures All authors declare no financial support or relationships that may pose conflict of interest.

\section{CHEMOTHERAPY DURING PREGNANCY AND NEONATAL OUTCOME: A RETROSPECTIVE ANALYSIS ON 47 PATIENTS}

${ }^{1}$ Eugenia DI Loreto, ${ }^{2}$ Alessandra Familiari, ${ }^{2}$ Veronica Accurti, ${ }^{3}$ Tiziana Boggini, ${ }^{1}$ Carlotta Castellani, 'Gianpiero Polverino, ${ }^{4}$ Julia Bewart, ${ }^{3}$ Monica Fumagalli, ${ }^{4}$ Fedro Alessandro Peccatori, 'Giovanna Scarfone. 'Fondazione Irccs Ca' Granda - Ospedale Maggiore Policlinico; Department of Gynecology; ${ }^{2}$ Fondazione Irccs Ca' Granda - Ospedale Maggiore Policlinico; Department of Maternal-Fetal Medicine; ${ }^{3}$ Fondazione Irces Ca' Granda - Ospedale Maggiore Policlinico; Neonatal Intensive Care Unit; ${ }^{4}$ European Institute of Oncology (leo); Department of Gynecology

\subsection{6/ijgc-2020-ESG0.85}

Introduction/Background The maternal diagnosis of cancer complicates approximately $0,1 \%$ of all pregnancies. The most frequently diagnosed malignancies are breast cancer, cervical cancer, lymphoma, ovarian cancer and melanoma. Although chemotherapy can be administered during pregnancy, its effects on obstetric and neonatal outcomes are still largely unknown. The aim of this study is to assess the oncologic management as well as the obstetric and perinatal outcomes in a consecutive series of patients diagnosed with cancer during pregnancy. Methodology Retrospective cohort study including 47 pregnant women diagnosed with primary invasive cancer during pregnancy between 2010 and 2019 at IRCCS Policlinico di Milano and Istituto Europeo Di Oncologia (Milan, Italy). All the included patients have been treated with chemotherapy during pregnancy. Oncologic, obstetric and neonatal data have been collected and compared. Linear regression analysis was used to assess the correlation between the therapy and perinatal outcomes. All the analysis were performed with Stat Direct 2.7.9 (StatsDirect Ltd, Altrincham).

Results The most common malignancies diagnosed in our cohort of 47 women were: breast cancer as the most common type $(87,2 \%)$, followed by lymphomas $(6,4 \%)$. All the maternal characteristics are listed in table 1.
Abstract 389 Table 1 Baseline demographics of the study population

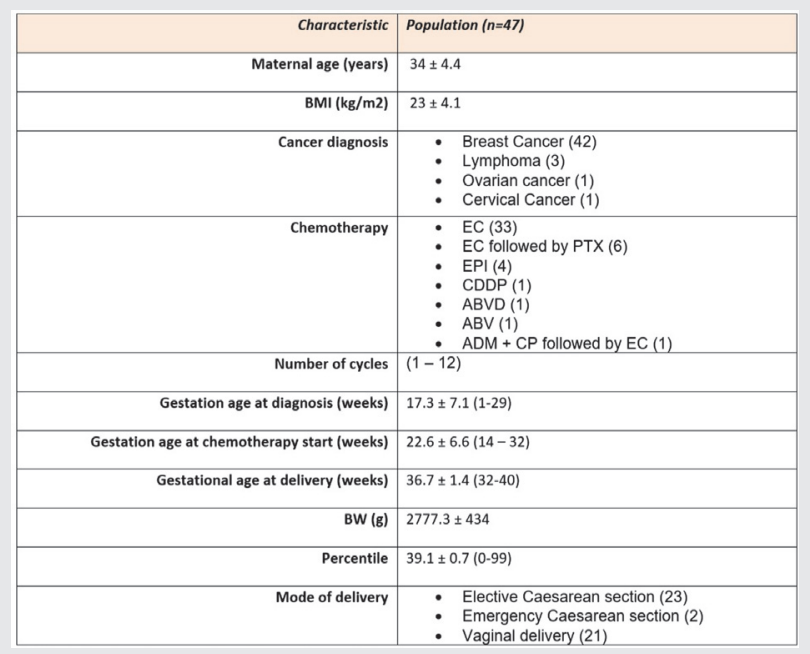

Data are given as mean \pm SD or $\mathrm{n}(\%)$.

$\mathrm{BW}=$ Birthweight, $\mathrm{EC}=$ Epirubicin and cyclophosphamide, PTX=Paclitaxel, CDDP=Cisplatin $C P=$ Cyclophosphamide, $A D M=A d r i a m y c i n, A B V D=A d r i a m y c i n, B l e o m y c i n, D a c a r b a z i n e$, Vinblastine

Abstract 389 Table 2 Results of the linear regression analysis of factors associated with neonatal outcomes

\begin{tabular}{|l|l|l|l|l|l|}
\hline & $\begin{array}{l}\text { BW percentile } \\
P \text { value }\end{array}$ & $\begin{array}{l}\text { 1st minute Apgar } \\
\text { P value }\end{array}$ & $\begin{array}{l}\text { 5th minute Apgar } \\
P \text { value }\end{array}$ & $\begin{array}{l}\text { Neonatal Hb } \\
P \text { value }\end{array}$ & $\begin{array}{l}\text { Neonatal WBC } \\
P \text { value }\end{array}$ \\
\hline$N^{\circ}$ of cycles & 0.63 & 0.28 & 0.18 & 0.88 & 0.77 \\
\hline GA at start & 0.90 & 0.30 & 0.62 & 0.75 & 0.83 \\
\hline
\end{tabular}

BW=Birthweight; $\mathrm{Hb}=$ Haemoglobin, $\mathrm{WBC}=$ White Blood Cells

All the patients were treated with chemotherapy during pregnancy consisting of different number of cycles according to the gestational age at diagnosis $(1-12)$. Maternal chemotherapy-related toxicity was generally lower than expected, maximum grade 1 according to National Cancer Institute Common toxicity Criteria (NCI-CTC).

All pregnancies ended with a livebirth fetus, at a mean gestational age of 36,7 weeks. The delivery was planned at least 3 weeks after the last administration of chemotherapy: 21 patients had a vaginal delivery, 23 an elective caesarean section and 2 an emergency caesarean section.

Table 2 shows the results of the correlation between maternal chemotherapy and perinatal outcomes: neonatal birthweight percentile, Apgar score and blood count parameters. No correlation has been demonstrated neither for the number of cycles or the gestational age at diagnosis and any of the outcome considered.

Conclusion Our results show that administration of chemotherapy during pregnancy is not associated with perinatal complications. Neonatal birth weight, Apgar score and neonatal hematologic indices are not affected by the number of chemotherapy cycles and the gestational age at the beginning of the treatment.

Disclosures Nothing to disclose. 